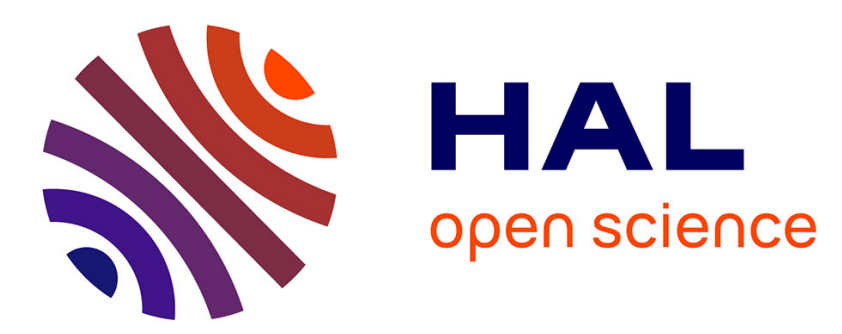

\title{
Decentralized Multi-Charger Coordination for Wireless Rechargeable Sensor Networks
}

Lei Mo, Pengcheng You, Xianghui Cao, Ye-Qiong Song, Jiming Chen

\section{To cite this version:}

Lei Mo, Pengcheng You, Xianghui Cao, Ye-Qiong Song, Jiming Chen. Decentralized Multi-Charger Coordination for Wireless Rechargeable Sensor Networks. 34th IEEE - International Performance Computing and Communications Conference, Dec 2015, Nanjing, China. hal-01241224

\section{HAL Id: hal-01241224 \\ https://hal.inria.fr/hal-01241224}

Submitted on 10 Dec 2015

HAL is a multi-disciplinary open access archive for the deposit and dissemination of scientific research documents, whether they are published or not. The documents may come from teaching and research institutions in France or abroad, or from public or private research centers.
L'archive ouverte pluridisciplinaire $\mathbf{H A L}$, est destinée au dépôt et à la diffusion de documents scientifiques de niveau recherche, publiés ou non, émanant des établissements d'enseignement et de recherche français ou étrangers, des laboratoires publics ou privés. 


\title{
Decentralized Multi-Charger Coordination for Wireless Rechargeable Sensor Networks
}

\author{
Lei Mo* ${ }^{* \ddagger}$ Pengcheng You*, Xianghui Cao ${ }^{\dagger}$, Ye-Qiong Song ${ }^{\ddagger}$ and Jiming Chen* \\ ${ }^{*}$ Department of Control Science and Engineering, Zhejiang University \\ Email: \{pcyou, xhcao,jmchen\}@iipc.zju.edu.cn \\ ${ }^{\dagger}$ School of Automation, Southeast University \\ Email: xhcao@seu.edu.cn \\ ${ }^{\ddagger}$ LORIA, Université de Lorraine \\ Email: \{lei.mo, ye-qiong.song $\} @ 1$ loria.fr
}

\begin{abstract}
Wireless charging is a promising technology for provisioning dynamic power supply in wireless rechargeable sensor networks (WRSNs). The charging equipment can be carried by some mobile nodes to enhance the charging flexibility. With such mobile chargers (MCs), the charging process should simultaneously address the MC scheduling, the moving and charging time allocation, while saving the total energy consumption of MCs. However, the efficient solutions that jointly solve those challenges are generally lacking in the literature. First, we investigate the multi-MC coordination problem that minimizing the energy expenditure of MCs while guaranteeing the perpetual operation of WRSNs, and formulate this problem as a mixed-integer linear program (MILP). Second, to solve this problem efficiently, we propose a novel decentralized method which is based on Benders decomposition. The multi-MC coordination problem is then decomposed into a master problem (MP) and a slave problem (SP), with the MP for MC scheduling and the SP for MC moving and charging time allocation. The MP is being solved by the base station (BS), while the SP is further decomposed into several sub-SPs and being solved by the MCs in parallel. The BS and MCs coordinate themselves to decide an optimal charging strategy. The convergence of proposed method is analyzed theoretically. Simulation results demonstrate the effectiveness and scalability of the proposed method.
\end{abstract}

Index Terms-Wireless rechargeable sensor networks, mobile charger coordination, perpetual operation, mixed-integer linear program, decentralized method

\section{INTRODUCTION}

Recently, due to the rapid development of wireless energy transfer technology [1], [2], wireless rechargeable sensor networks (WRSNs) have become a hot research topic [3]-[7]. Unlike the traditional wireless sensor networks (WSNs), WRSNs can avoid the limitations introduced by the energy-constrained sensor nodes, and make the network lifetime extend to infinitely. To achieve this goal, the charging behavior of MCs becomes a key design issue.

The traveling salesman problem (TSP)-based [3], [8]-[10] and orienteering problem (OP)-based [11] methods are the two most popular methods for solving charging optimization problems. The basic idea is to model a WRSN as a graph, where the vertexes and the edges represent the sensor nodes and the distances between them, respectively. The vertex usually associates with charging profit, e.g., the replenished energy or charging emergency, while the edge usually associates with charging cost, e.g., the traveling time or traveling energy. Then the aim of optimization problems is to maximize the charging profits, or minimize the charging costs, under the constraints of visiting all the vertexes.
However, the limitations of those methods are that they can only handle the single-MC charging problem. To enhance the system reliability and scalability, introducing multi-MC is desirable. For the multi-MC case, multiple traveling salesman problem (MTSP)-based or team orienteering problem (TOP)-based methods can be used to handle the charging optimization problem [5], [12]. However, when taking multiple requirements such as the perpetual operation, MC charging ability, or enhance energy efficiency into account, the optimization problems will become very difficult to solve, no matter the single-MC or multi-MC cases. To solve those complex optimization problems, there have two common methods. The first one is to simplify the problem [4], [5], [13], i.e., under specific conditions or assumptions, transfer the original problem to a standard TSP/MTSP. While the second one is to approximate/relax the original problem to a nonlinear problem (NLP) [3], [9]-[11], or to solve the problem through the heuristic/adaptive methods [8]. However, those methods are hard to get an optimal solution, and their computational complexity is relatively high. Moreover, most of the existing methods are centralized. Basically, an BS is needed to collect the sensor information periodically, calculate the charging strategy, and send the control commands to the MCs. However, the centralized architecture limits the applicability in a large scale networks. In order to enhance the system scalability and reliability, as well as make a better usage of network resources, decentralized method should be introduced to coordinate multi-MC.

In this paper, we study the multi-MC coordination problem to fulfil the sensor charging task. The primary goal is to achieve the perpetual operation. On this basis, we aim to minimize the total energy consumption of MCs, including their traveling and charging costs. Hence, the scheduling, the moving and charging time allocation of MCs are the optimization objectives. During the design, we need to face the following questions: First, what is the condition for each sensor node never running out of its energy? Second, if this is possible, how about the order to charge those sensor nodes, and which sensor node a MC should select to charge such that the total energy consumption of MCs is minimized? Third, is there a way to achieve optimal solution while avoiding high computational complexity?

The available methods are hard to handle above problems simultaneously. Although the first and the second problems mentioned above have been partially studied in some works, e.g., [3], [8], [9], the joint-design studies of the multi-MC scheduling, the 
moving and charging time allocation, and the energy efficiency are very rare, especially considering the decentralized coordination, which is important to enhance the system scalability and reliability. To this end, in this paper, we propose a novel multiMC coordination method for WRSNs. We first formulate the multi-MC coordination problem as a MILP problem, which is NP-hard in general [14]. By employing Benders decomposition [15] and dual decomposition [16], we propose a decentralized method to achieve optimal solution, while balancing the system performance and computational complexity. To the best of our knowledge, this is the first work to address the decentralized multi-MC scheduling and charging joint-design problem.

Our main contributions focus on how to formulate the jointdesign problem properly and how to solve this problem efficiently, which can be summarized as follows:

1) We propose a novel multi-MC coordination framework for WRSNs through optimizing the MC scheduling, the moving and charging time allocation, and the energy efficiency problems jointly. More importantly, we adopt a decentralized manner to coordinate multi-MC, and hence reducing the computational complexity and enhancing the system scalability greatly.

2) To enhance the charging efficiency, based on the energy consumption model of the sensor nodes and the charging model of the MCs, we derive a lifetime-based charging order and divide the sensor nodes into several groups. On this basis, we provide a sufficient condition to keep system operating perpetually. Taking this constraint into account, as well as the practical requirements such as the moving and charging abilities of the MCs, and with an objective to save their energy consumptions, we then formulate the joint optimization problem as a MILP problem.

3) Through analyzing the characteristics of MILP problem, we propose a Benders-based decentralized method to solve this problem. This method decomposes the MILP problem into a MP for the MC scheduling problem, and a SP for the MC moving and charging time problem. On this basis, the SP is further decomposed into several sub-SPs and can be assigned to proper MCs. Through the coordination between BS and MCs, we can derive an optimal charging strategy. Finally, we provide a sufficient condition to make the solution converge.

The remainder of this paper is organized as follows. Section II presents the system model and formulates the problem. Then the decentralized multi-MC coordination mechanism is designed in Section III. Finally, Section IV shows the simulation results and Section V concludes this paper.

\section{System AND PRoblem Formulation}

In this section, we shall first present the system model of WRSNs. We then formulate the multi-MC coordination problem as a MILP problem which takes the perpetual operation, energy efficiency, and MC charging ability into account.

\section{A. System model}

1) Overview: Considering a WRSN contains $n$ sensor nodes $\left\{s_{1}, \ldots, s_{n}\right\}$, we are interested in charging those nodes using $m$ MCs $\left\{c_{1}, \ldots, c_{m}\right\}$, as shown in Fig. 1. Each sensor node

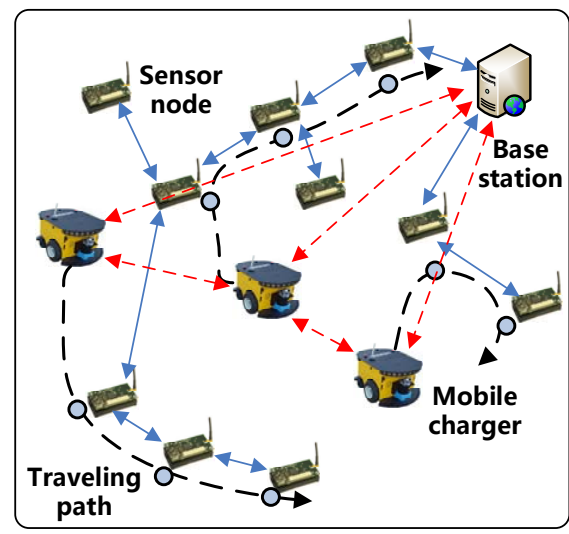

Fig. 1. System framework.

has a battery with maximum capacity $e_{\max }$. When $s_{i}$ 's residual energy $e_{i}$ is lower than $e_{\min }, s_{i}$ will stop working. Moreover, $s_{i}$ reports its $e_{i}$ periodically to the BS through the single or multihop communications. Based on the collected information, the BS coordinates with the MCs to make the charging decisions, and then, the MCs follow this decision to move and perform the charging tasks. During this process, the MCs follow a periodic scheduling: In each cycle, they start from the BS, travel at a speed of $v \mathrm{~m} / \mathrm{s}$, and return to the BS for the battery charging or replacement (denote this time as $\tau_{s}$ ).

2) Energy consumption model: For the sensor node, since the data communication (transmission and reception) is the most energy consuming part, we consider the following energy consumption model [3]:

$$
r_{i}^{t}=\rho \sum_{k=1, k \neq i}^{n} f_{k i}+\sum_{j=1, j \neq i}^{n} C_{i j} f_{i j}+C_{i b} f_{i b}, \quad \forall i,
$$

where $r_{i}^{t}$ is the energy consumption rate of $s_{i}, f_{i j}$ and $f_{i b}$ are the flow rate from $s_{i}$ to $s_{j}$, and $s_{i}$ to BS, respectively. $\rho$ and $C_{i j}$ (or $C_{i b}$ ) are the rate of energy consumption for receiving a unit of data rate, and transmitting a unit of data rate from $s_{i}$ to $s_{j}$ (or the BS), respectively. To simplify the problem, we assume the energy consumption rate of a sensor node is invariant with time.

3) Energy charging model: For the typical wireless rechargeable tag such as Intel Research's wireless identification and sensing platform (WISP), the receiving power is a decreasing function of distance from the charger, which can be described by the following model [17]:

$$
p_{r}=\frac{G_{s} G_{r} \iota}{L_{p}}\left[\frac{\omega}{4 \pi(d+\zeta)}\right]^{2} p_{0},
$$

where $d$ is the distance between the sensor node and MC, $p_{0}$ is the source power, $G_{s}$ is the source antenna gain, $G_{r}$ is the receive antenna gain, $L_{p}$ is the polarization loss, $\omega$ is the wavelength, $\iota$ is the rectifier efficiency, and $\zeta$ is a parameter to adjust the Friis' free space equation for short distance transmission.

(2) shows that the charging efficiency declines exponentially as the distance $d$ increases. As the experiments show in [4], when an $\mathrm{MC}$ is placed $10 \mathrm{~cm}$ away from a sensor node, the charging efficiency reduces to $1.5 \%$. Therefore, we assume the MC start charging when it arrives at a sensor node, i.e., $d \rightarrow 0$. Hence, (2) reduces to

$$
p_{r}=\varsigma p_{0},
$$


where $\varsigma=\frac{G_{s} G_{r} \iota \omega^{2}}{16 L_{p} \pi^{2} \zeta^{2}}$ is the charging efficiency.

\section{B. Problem formulation}

\section{1) Charging sequence determination:}

Definition 2.1: The network exists perpetual operation if it meets the following requirement: the energy level of sensor node $s_{i}, \forall i$ will never fall below $e_{\min }$.

Definition 2.2: When $N(N \leq n)$ sensor nodes requiring charging, define the renewable cycle as the time to charge $N$ sensor nodes once, while the charging round as the time to charge $m$ sensor nodes once. Hence, one renewable cycle may contain several charging rounds.

Since different sensor nodes have different residual energies and energy consumption rates, for $s_{i}$, to evaluate its charging priority, we can use $s_{i}$ 's lifetime

$$
L_{i}=\frac{e_{i}^{0}-e_{\min }}{r_{i}^{t}}, \forall i,
$$

as an index, where $e_{i}^{0}$ is the initial energy of $s_{i}$ in current renewable cycle.

To put the sensor nodes in a proper charging order, we sort all the nodes according to their lifetimes in an ascending order:

$$
\boldsymbol{L}=\left\{L_{1}, L_{2}, \ldots, L_{n}\right\} .
$$

Note that in (5), the subscripts of $\left\{L_{1}, \ldots, L_{n}\right\}$ have been rearranged such that $L_{1} \leq L_{2} \leq \ldots \leq L_{n}$.

To enhance charging efficiency, in each renewable cycle, there is no need to charge all the nodes since some of them have enough energy to survive until get charged in the next renewable cycle. Through the following Proposition, we can divided $n$ sensor nodes into two sets: the serving sensor set $\mathcal{N}$, and the non-serving sensor set $\overline{\mathcal{N}}$, where the nodes in $\mathcal{N}$ have to be charged in the current renewable cycle, while the others, i.e., the nodes in $\overline{\mathcal{N}}$, can be charged in the next renewable cycle.

Proposition 2.1: For $s_{i}$, if $L_{i}$ satisfies

$$
L_{i} \geq(2 r-1) \varrho+\tau_{s},
$$

$s_{i} \in \overline{\mathcal{N}}$, else, $s_{i} \in \mathcal{N}$, where $r=\left\lceil\frac{n}{m}\right\rceil,\lceil\cdot\rceil$ is a ceiling operator, $\varrho=\frac{e_{\max }-e_{\min }}{p_{r}}+\frac{d_{\max }}{v}, d_{\max }$ is the maximum distance between any two sensor nodes.

Assume that $\mathcal{N}$ has $h$ sensor nodes, where $h \leq n$, and $t=\left\lceil\frac{h}{m}\right\rceil$. To charge the nodes in $\mathcal{N}$ effectively, it is reasonable to divide those nodes into several groups. Based on the property of charging efficiency, we assume one MC charges one sensor at a time. Note that the network has $m$ MCs, then in each round, $m$ sensor nodes can be charged simultaneously. Hence, we can derive a grouped list that contains $h$ shortest-lifetime sensor nodes

$$
\boldsymbol{L}_{s}=\{\underbrace{L_{1}, \ldots, L_{m}}_{\mathcal{R}_{1}}, \underbrace{L_{m+1}, \ldots, L_{2 m}}_{\mathcal{R}_{2}}, \ldots, \underbrace{L_{m(t-1)+1}, \ldots, L_{h}}_{\mathcal{R}_{t}}\},
$$

and schedule the MCs following the order $\left\{\mathcal{R}_{1}, \mathcal{R}_{2}, \ldots, \mathcal{R}_{t}\right\}$ to charge the sensor nodes.

Remark 2.1: The advantages of sensor node partition are twofold: the emergent handling ability can be enhanced while the computational complexity can be reduced. For example, when charging sensor nodes in $\mathcal{R}_{l}$, if sensor node $s_{j}, s_{j} \in \overline{\mathcal{N}}$ requires charging abruptly, based on $L_{j}$, we can put $s_{j}$ in the proper place of $\left\{\mathcal{R}_{l+1}, \mathcal{R}_{l+2}, \ldots\right\}$. Moreover, for an optimization problem, the computational complexity increases significantly with the number of variables and constraints, solving those smaller problems iteratively can be more efficient than solving a single large problem.

2) Charging optimization: Note that in the last charging round $\mathcal{R}_{t}$, the number of MCs may be larger than the sensor nodes. Without loss of generality, we assume that in each round, $m$ MCs are scheduled to charge $\tilde{n}$ sensor nodes, where $\tilde{n} \leq m$. Suppose that the current round is $\mathcal{R}_{l}$, the traveling and charging time in the previous $l-1$ rounds is $\tau_{l-1}$. To design the MC scheduling scheme, we introduce a $\tilde{n} \times m$ matrix $\boldsymbol{q}^{l}$, where $q_{i j}^{l}=$ 1 represents $c_{j}$ is scheduled to charge $s_{i}$, and $q_{i j}^{l}=0$, otherwise. Since in each charging round: (a) every $\mathrm{MC}$ is responsible for at most one sensor node, and (b) every sensor node is charged by one $\mathrm{MC}$, we can easily derive the following constraints:

$$
\begin{aligned}
& \sum_{i=1}^{\tilde{n}} q_{i j}^{l} \leq 1, \quad \forall j, \\
& \sum_{j=1}^{m} q_{i j}^{l}=1, \quad \forall i .
\end{aligned}
$$

On the other hand, to determine the charging time of MCs, we introduce a $\tilde{n} \times m$ matrix $\boldsymbol{t}^{l}$, where $t_{i j}^{l}$ represents the time for $c_{j}$ to charge $s_{i}$. To enhance the charging efficiency, we assume $c_{j} \mathrm{u}-$ tilizes its maximum power $p_{j}$ to perform the charging task. Based on the previous charging decisions $\left\{\boldsymbol{q}^{1}, \boldsymbol{t}^{1}, \ldots, \boldsymbol{q}^{l-1}, \boldsymbol{t}^{l-1}\right\}$, the residual energy of $c_{j}$ in the $l$ th round is

$$
E_{j}^{l}=E_{j}^{0}-\sum_{k=1}^{l-1} \sum_{i=1}^{\tilde{n}}\left(q_{i j}^{k} d_{i j}^{k} \epsilon+t_{i j}^{k} p_{j}\right), \quad \forall j,
$$

where $E_{j}^{0}$ is the initial energy of $c_{j}$ in current renewable cycle, $d_{i j}^{k}$ is the distance between $s_{j}$ in $\mathcal{R}_{k-1}$ and $s_{i}$ in $\mathcal{R}_{k}, \epsilon$ is the energy consumed by $c_{j}$ to move one unit distance.

It is obviously that $t_{i j}^{l}$ is related to $q_{i j}^{l}$ and $E_{j}^{l}$. Hence, we have

$$
0 \leq t_{i j}^{l} \leq k E_{j}^{l} q_{i j}^{l}, \quad \forall i, \quad \forall j,
$$

where $k$ is a positive constant and can be easily derived through the simulation/experiment.

To determine the amount of energy replenished to a sensor node, we should know how long this node can survive after being charged. For $s_{i} \in \mathcal{R}_{l}$, the worst case happens when $s_{i}$ is charged at the end of the next renewable cycle. Hence, the maximum waiting time is $(t-l+r) \varrho+\tau_{s}$. Since at the $l$ th round, $s_{i}$ has already consumed $e_{i}^{0}-\tau_{l-1} r_{i}^{t}$ energy. To achieve perpetual operation, the replenished energy of $s_{i}$ should no less than

$$
\underline{E}_{i}=\left[(t-l+r) \varrho+\tau_{s}\right] r_{i}^{t}-\left(e_{i}^{0}-\tau_{l-1} r_{i}^{t}\right), \quad \forall i .
$$

On the other hand, since the maximum battery capacity of $s_{i}$ is $e_{\max }$. The replenished energy of $s_{i}$ should not exceed

$$
\bar{E}_{i}=e_{\max }-\left(e_{i}^{0}-\tau_{l-1} r_{i}^{t}\right), \quad \forall i .
$$

Through (12) and (13), we can easily derive a proper range of energy replenished to $s_{i}$ :

$$
\underline{E}_{i} \leq \sum_{j=1}^{m} p_{j} t_{i j}^{l} \varsigma \leq \bar{E}_{i}, \quad \forall i .
$$

Here, we assume $\underline{E}_{i} \leq \bar{E}_{i}$. 
Remark 2.2: Since the MC movement is time consuming, when MCs arrive at their working points and start charging the sensor nodes, the residual energies of sensor nodes may have changed. However, the sensor nodes are usually low power, e.g., Mica2 node equipped with two AA batteries and can continue working 172 hours [18]. Since the moving time of MC in one charging round is much smaller than node's lifetime, the energy depletion of sensor node during the MC movement can be omitted here.

Note that constraint (14) is not the only sufficient condition to keep system operating perpetually. This is because during the energy estimation, we assume the maximum waiting time of $s_{i}$ is $(t-l+r) \varrho+\tau_{s}$, which implies there is no gap between two adjacent charging rounds. Hence, we introduce a $\tilde{n} \times m$ matrix $\boldsymbol{g}^{l}$ to bound the moving time, where $g_{i j}^{l}$ represents the time for MC moving from $s_{j}\left(s_{j} \in \mathcal{R}_{l-1}\right)$ to $s_{i}\left(s_{i} \in \mathcal{R}_{l}\right)$. It is obviously that $g_{i j}^{l}$ has a lower bound:

$$
g_{i j}^{l} \geq q_{i j}^{l} \frac{d_{i j}^{l}}{v}, \quad \forall i, \quad \forall j .
$$

To achieve the perpetual operation, $g_{i j}^{l}$ should not exceed a threshold. Denote this threshold as $\theta^{l}$, and we introduce the following constrain to restrict the charging and moving time in one charging round:

$$
0 \leq t_{i j}^{l}+g_{i j}^{l} \leq \theta^{l}, \quad \forall i, \quad \forall j,
$$

where the value of $\theta^{l}$ can be determined through the following Proposition.

Proposition 2.2: To ensure there is no gap between two adjacent charging rounds, we can select

$$
\theta^{l}=\min \left\{\varrho, \varpi_{l+1}^{l}, \ldots, \varpi_{t}^{l}\right\}
$$

where

$\varpi_{l+j}^{l}=L_{m(l+j-1)+1}-\tau_{l-1}-\sum_{i=1}^{j+1} \varrho-\frac{d_{\max }}{v}, 1 \leq j \leq t-l$.

Note that our goal is to achieve the perpetual operation, which can be guaranteed by the following Theorem.

Theorem 2.1: The sufficient condition to achieve the perpetual operation is that the constraints (14), (15), and (16) must be satisfied.

Taking the above constraints into account, the optimization problem can be formulated as follows:

\section{Multi-MC coordination problem}

$$
\begin{array}{cl}
\min _{\boldsymbol{q}^{l}, \boldsymbol{t}^{l}, \boldsymbol{g}^{l}} & \Phi=\sum_{i=1}^{\tilde{n}} \sum_{j=1}^{m}\left(q_{i j}^{l} d_{i j}^{l} \epsilon+p_{j} t_{i j}^{l}\right), \\
\text { s.t. } & \text { (8), (9), (11), (14), (15), (16). }
\end{array}
$$

Problem (19) is a MILP problem since it contains three types of constraints: the integer constraints (8) and (9); the continuous constraints (14) and (16); and the mixed constraints (11) and (15).

\section{CHARGER COORDINATION}

In this section, based on the characteristics of problem (19), we design an novel multi-MC coordination mechanism. In order to reduce computational complexity as well as to enhance the system scalability and reliability, the coordination mechanism runs in a decentralized manner.

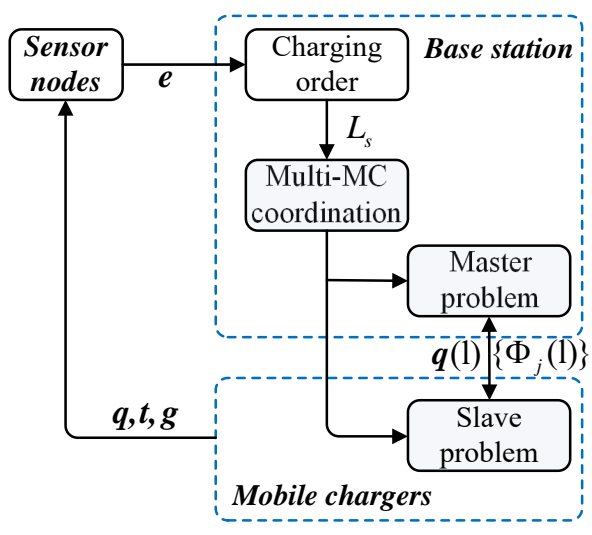

Fig. 2. Structure of decentralized implementation.

\section{A. Benders-based decentralized coordination}

To solve problem (19) efficiently, finding an optimal $\boldsymbol{q}^{l}$ is the most important part, since if $\boldsymbol{q}^{l}$ is determined, problem (19) will reduce to a linear program (LP) problem, which has a simpler structure, and easier to solve. Benders decomposition [19] is an effective method for solving certain classes of mixed optimization problem such as MILP. The basic idea is decomposing the MILP problem into a master problem (MP) and a slave problem (SP), where the MP only contains the integer constraints, while the other constraints are considered in the SP. According to the iterations between the MP and SP, we can derive an optimal solution. The algorithm structure is shown in Fig. 2. For simplicity and generality, we drop round index here. Based on the structure of Benders decomposition, the MP and $\mathrm{SP}$ can be formulated as follows.

MP

$$
\begin{aligned}
& \Phi_{\text {lower }}=\min _{\boldsymbol{q}} \Phi, \\
& \text { s.t. }\left\{\begin{array}{l}
\sum_{i=1}^{\tilde{n}} q_{i j} \leq 1, \quad \forall j, \\
\sum_{j=1}^{m} q_{i j}=1, \quad \forall i, \\
\text { feasibility constraints, } \\
\text { infeasibility constraints. }
\end{array}\right.
\end{aligned}
$$

Comparing problem (20) with problem (19), we can see that the constraints are loosened. Hence, $\Phi_{\text {lower }}$ is a lower bound of $\Phi$. The feasibility and infeasibility constraints are come from the solution to the SP, which help to narrow the search region of integer variables, and their forms will be shown in (28) and (29), respectively.

$$
\begin{aligned}
& \text { SP } \\
& \Phi_{\text {upper }}=\min _{\boldsymbol{t}, \boldsymbol{g}} \Phi=\sum_{i=1}^{\tilde{n}} \sum_{j=1}^{m}\left(q_{i j}(l) d_{i j} \epsilon+p_{j} t_{i j}\right), \\
& \text { s.t. }\left\{\begin{array}{l}
0 \leq t_{i j} \leq k E_{j} q_{i j}(l), \quad \forall i, \quad \forall j, \\
\underline{E}_{i} \leq \sum_{j=1}^{m} p_{j} t_{i j} \varsigma \leq \bar{E}_{i}, \quad \forall i, \\
g_{i j} \geq q_{i j}(l) \frac{d_{i j}}{v}, \quad \forall i, \quad \forall j, \\
0 \leq t_{i j}+g_{i j} \leq \theta, \quad \forall i, \forall j,
\end{array}\right.
\end{aligned}
$$

where $\boldsymbol{q}(l)$ is given by solving problem (20) at the $l$ th iteration. Since (a) $\boldsymbol{q}(l)$ may be just a feasible solution (not optimal yet), and (b) our aim is to minimize the objective function, $\Phi_{\text {upper }}$ is an upper bound of $\Phi$. 
Denote $\Phi^{*}$ as the optimal value of $\Phi$. From problems (20) and (21), we can see that $\Phi^{*}$ lies between $\Phi_{\text {lower }}$ and $\Phi_{\text {upper. }}$. Through the iterations between problems (20) and (21), the gap between $\Phi_{\text {lower }}$ and $\Phi_{\text {upper }}$ will be gradually reduced and finally we can get the optimal solution $\Phi^{*}$. The iteration process of Benders-based decentralized method can be summarized as follows:

\section{Step 1: Initialization}

Initialize the iteration counter $l=0$, the solution $\boldsymbol{q}(0)$ to the MP, the lower bound of objective function $\Phi_{\text {lower }}=-\infty$, and its upper bound $\Phi_{\text {upper }}=\infty$. The feasibility and infeasibility constraints are set to null.

\section{Step 2: SP solution}

Since without the integer variable $\boldsymbol{q}$ coupling the MCs together, problem (21) can be decoupled into $m$ sub-SPs for the $\mathrm{MCs}$, and hence each sub-SP can be solved by correspond MC in parallel.

Sub-SP

$$
\begin{array}{ll}
\min _{\boldsymbol{t}, \boldsymbol{g}} & \Phi_{j}=\sum_{i=1}^{\tilde{n}}\left(q_{i j}(l) d_{i j} \epsilon+p_{j} t_{i j}\right), \\
\text { s.t. } & (11),(14),(15),(16) \text { for given } \boldsymbol{q}(l) .
\end{array}
$$

Introducing the Lagrangian multipliers $\boldsymbol{\lambda}(l) \geq 0, \boldsymbol{\mu}(l) \geq 0$, $\boldsymbol{\alpha}(l) \geq 0, \boldsymbol{\beta}(l) \geq 0, \boldsymbol{\gamma}(l) \geq 0, \boldsymbol{\rho}(l) \geq 0, \varepsilon(l) \geq 0$, the Lagrangian to problem (22) is

$$
\begin{aligned}
\mathcal{L}_{j}= & \sum_{i=1}^{\tilde{n}}\left[\left(q_{i j}(l) d_{i j} \epsilon+p_{j} t_{i j}\right)-t_{i j} \lambda_{i j}(l)\right. \\
& +\left(t_{i j}-k E_{j} q_{i j}(l)\right) \mu_{i j}(l)+\left(\underline{E}_{i} q_{i j}(l)-p_{j} t_{i j} \varsigma\right) \alpha_{i j}(l) \\
& +\left(p_{j} t_{i j} \varsigma-\bar{E}_{i} q_{i j}(l)\right) \beta_{i j}(l)+\left(q_{i j}(l) \frac{d_{i j}}{v}-g_{i j}\right) \gamma_{i j}(l) \\
& \left.-\left(t_{i j}+g_{i j}\right) \rho_{i j}(l)+\left(t_{i j}+g_{i j}-\theta\right) \varepsilon_{i j}(l)\right] \\
= & \sum_{i=1}^{\tilde{n}}\left(\mathcal{F}_{i j} t_{i j}+\mathcal{P}_{i j} g_{i j}\right)+\mathcal{D}_{j}
\end{aligned}
$$

where

$$
\begin{aligned}
\mathcal{F}_{i j}= & p_{j}-\lambda_{i j}(l)+\mu_{i j}(l)-p_{j} \varsigma \alpha_{i j}(l)+p_{j} \varsigma \beta_{i j}(l)-\rho_{i j}(l) \\
& +\varepsilon_{i j}(l), \\
\mathcal{P}_{i j}= & -\gamma_{i j}(l)-\rho_{i j}(l)+\varepsilon_{i j}(l), \\
\mathcal{D}_{j}= & \sum_{i=1}^{\tilde{n}}\left(q_{i j}(l) d_{i j} \epsilon-k E_{j} q_{i j}(l) \mu_{i j}(l)+\underline{E}_{i} q_{i j}(l) \alpha_{i j}(l)\right. \\
& \left.-\bar{E}_{i} q_{i j}(l) \beta_{i j}(l)+q_{i j}(l) \frac{d_{i j}}{v} \gamma_{i j}(l)-\theta \varepsilon_{i j}(l)\right) .
\end{aligned}
$$

Based on the definition of duality [16], problem (22) has the following dual problem:

Dual Sub-SP

$$
\begin{aligned}
& \max _{\substack{\boldsymbol{\lambda}(l), \boldsymbol{\mu}(l), \boldsymbol{\alpha}(l), \boldsymbol{\beta}(l), \boldsymbol{\gamma}(l), \boldsymbol{\rho}(l), \boldsymbol{\varepsilon}(l)}} \mathcal{D}_{j}, \\
& \text { s.t. } \quad\left\{\begin{array}{l}
\mathcal{F}_{i j} \geq 0, \mathcal{P}_{i j} \geq 0, \forall i, \\
\boldsymbol{\lambda}(l) \geq 0, \boldsymbol{\mu}(l) \geq 0, \boldsymbol{\alpha}(l) \geq 0, \\
\boldsymbol{\beta}(l) \geq 0, \gamma(l) \geq 0, \boldsymbol{\rho}(l) \geq 0, \boldsymbol{\varepsilon}(l) \geq 0 .
\end{array}\right.
\end{aligned}
$$

Note that problem (22) is a LP problem, thus the strong duality is guaranteed [16], which means the optimal value of problem (27) is identical to that of problem (22). Solving problem (27) through the existing LP methods, we can derive the continuous variables $\boldsymbol{\lambda}(l), \boldsymbol{\mu}(l), \boldsymbol{\alpha}(l), \boldsymbol{\beta}(l), \boldsymbol{\gamma}(l), \boldsymbol{\rho}(l), \boldsymbol{\varepsilon}(l)$.

\section{Step 3: Convergence checking}

$\Phi_{\text {lower }}(l)$ is given by submitting $\boldsymbol{q}(l)$ into problem (20), while $\Phi_{\text {upper }}(l)=\sum_{j=1}^{m} \max \mathcal{D}_{j}$, and $\max \mathcal{D}_{j}$ is given by submitting $\boldsymbol{\lambda}(l), \boldsymbol{\mu}(l), \boldsymbol{\alpha}(l), \boldsymbol{\beta}(l), \boldsymbol{\gamma}(l), \boldsymbol{\rho}(l), \boldsymbol{\varepsilon}(l)$ into problem (27). Denote $\iota$ as a small tolerance. When $\left|\Phi_{\text {upper }}(l)-\Phi_{\text {lower }}(l)\right|<\iota$, the algorithm stops. Otherwise, the algorithm continues at the next step.

\section{Step 4: Master Problem Solution}

The iteration counter $l$ is increased, and the Benders cuts will be added into problem (20). Note that in this step, $\boldsymbol{q}(l+1)$ are the integer variables we want to calculate. According to the solution to problem (27), two different types of constraints can be added into problem $(20)$ at the $(l+1)$ th iteration.

1) If problem (27) is infeasible, then problem (22) has an unbounded solution, and problem (19) has no physical solution.

2) If problem (27) has a bounded solution, e.g., $\boldsymbol{\lambda}(l), \boldsymbol{\mu}(l)$, $\boldsymbol{\alpha}(l), \boldsymbol{\beta}(l), \gamma(l), \boldsymbol{\rho}(l), \boldsymbol{\varepsilon}(l)$. Through the duality, problem (22) has a feasible solution. However, $\mid \Phi_{\text {upper }}(l)-$ $\Phi_{\text {lower }}(l) \mid>\iota$ since $\boldsymbol{q}(l)$ is not an optimal solution. In order to reduce the gap between $\Phi_{\text {lower }}(l+1)$ and $\Phi_{\text {upper }}(l+1)$, at the $(l+1)$ th iteration, we add a feasibility constraint into problem (20) so as to make $\Phi_{\text {lower }}(l+1)>\Phi_{\text {lower }}(l)$.

3) If problem (27) has an unbounded solution, problem (22) has no feasible solution under the given $\boldsymbol{q}(l)$. At the $(l+1)$ th iteration, an infeasibility constraint should be added into problem (20) to avoid selecting those infeasible integers again.

When problem (20) is solved, Steps 2-3 are repeated. Algorithm 1 summarizes the implementation details of Benders-based decentralized multiple MCs coordination mechanism.

Theorem 3.1: The sufficient condition to make the solution of problem (27) converge is that the feasibility and infeasibility constraints added into problem (20) should be

$$
\begin{aligned}
& \sum_{i=1}^{\tilde{n}} \sum_{j=1}^{m}\left(q_{i j}(l+1) d_{i j} \epsilon+p_{j} t_{i j}(l)\right) \\
\geq & \sum_{i=1}^{\tilde{n}} \sum_{j=1}^{m}\left(q_{i j}(l+1) d_{i j} \epsilon-k E_{j} q_{i j}(l+1) \mu_{i j}(v)\right. \\
& +\underline{E}_{i} q_{i j}(l+1) \alpha_{i j}(v)-\bar{E}_{i} q_{i j}(l+1) \beta_{i j}(v) \\
& \left.+q_{i j}(l+1) \frac{d_{i j}}{v} \gamma_{i j}(v)-\theta \varepsilon_{i j}(v)\right), \quad \forall v \in \mathcal{A},
\end{aligned}
$$

and

$$
\begin{aligned}
& 0 \geq \sum_{i=1}^{p}\left[-k E_{i} q_{i j}(l+1) \mu_{i j}(\vartheta)+\underline{E}_{i} q_{i j}(l+1) \alpha_{i j}(\vartheta)\right. \\
&\left.-\bar{E}_{i} q_{i j}(l+1) \beta_{i j}(\vartheta)+q_{i j}(l+1) \frac{d_{i j}}{v} \gamma_{i j}(\vartheta)-\theta \varepsilon_{i j}(\vartheta)\right] \\
& \quad \forall \vartheta \in \mathcal{B}
\end{aligned}
$$

respectively, where $\mathcal{A}$ and $\mathcal{B}$ are the sets of iterations that problem (27) has the bounded and unbounded solutions, i.e., $\mathcal{A} \leftarrow l \cup \mathcal{A}$ if at the $l$ th interaction problem (27) has an bounded solution, $\mathcal{B} \leftarrow l \cup \mathcal{B}$ if at the $l$ th interaction problem (27) has an unbounded solution. 


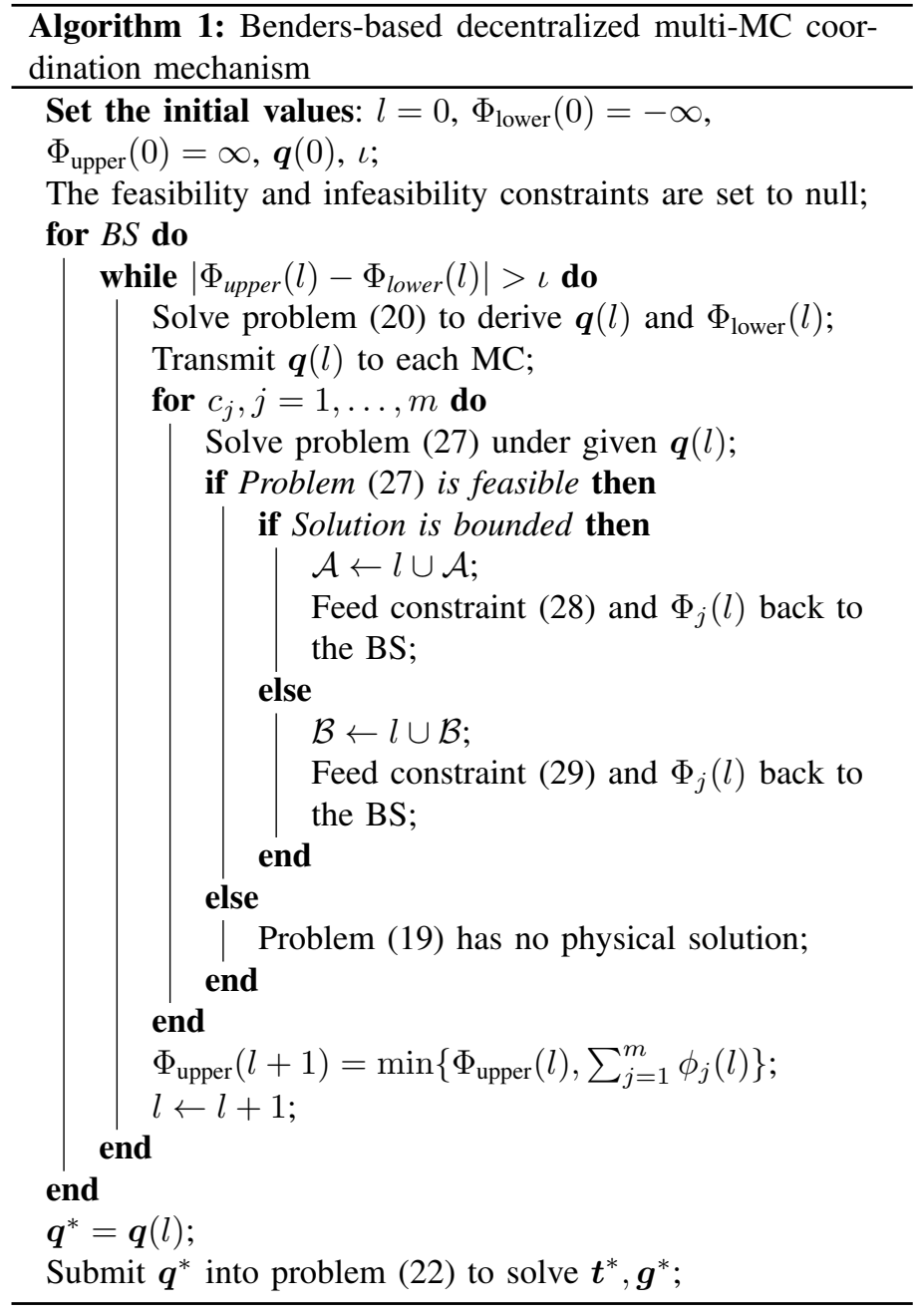

\section{Simulation}

We consider a WRSN containing 20 sensor nodes and 4 MCs. The energy consumption rate and initial energy of each sensor node are randomly generated between $[0.3,0.5] \mathrm{J} / \mathrm{s}$, and $[400,250] \mathrm{J}$, respectively. The network sampling period is $0.2 \mathrm{~s}$. For a sensor node, $e_{\max }=1000 \mathrm{~J}, e_{\min }=200 \mathrm{~J}$. The serving time, charging power, charging efficiency, traveling speed, and unit moving energy of $\mathrm{MC}$ are set to $\tau_{s}=3000 \mathrm{~s}, p_{j}=10 \mathrm{~W}$, $\varsigma=0.5, v=2 \mathrm{~m} / \mathrm{s}$, and $\epsilon=1 \mathrm{~J}$, respectively.

Fig. 3(a) shows the energy charging error in the first renewable cycle, where the energy charging error represents the mean square error (MSE) between the residual energy and desired energy, i.e., $R_{l}(k)=\sqrt{\frac{\sum_{i=1}^{4}\left(\sum_{j=1}^{m} p_{j} t_{i j}^{l} \varsigma-e_{i}(k)\right)^{2}}{4}}, l=1, \ldots, 5$, $e_{i}(k)$ is $s_{i}$ 's residual energy in step $k$. Through condition (6), all the sensor nodes require charging in the first renewable cycle. Hence, one cycle can be divided into five charging rounds. From Fig. 3(a), we can see that there is no gap between two adjacent charging rounds. Since without charging, $e_{i}$ will decrease with time. The earlier the MCs start next round charging, the lesser energies the sensor nodes should be replenished.

Fig. 3(b) shows the energy behavior of 20 sensor nodes in five renewable cycles. The vertical coordinate represents the MSE between the residual and minimum working energies, i.e.,

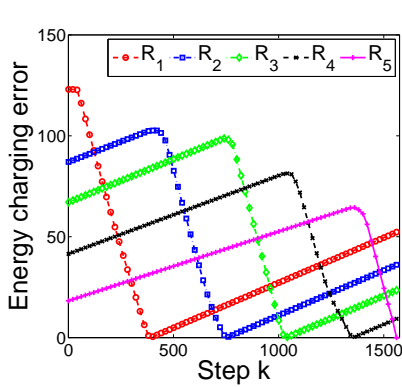

(a)

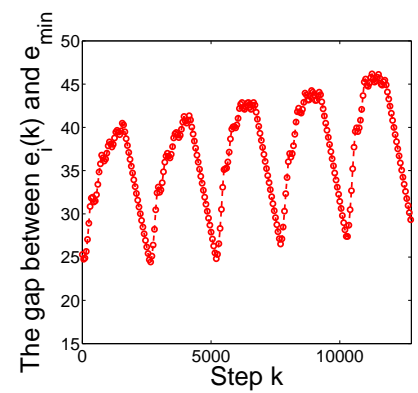

(b)
Fig. 3. a) The energy charging error of 20 sensor nodes in the first renewable cycle. b) The energy behavior of 20 sensor nodes in five renewable cycles.

$\sqrt{\frac{\sum_{i=1}^{20}\left(e_{i}(k)-e_{\min }\right)^{2}}{20}}$. From Fig. 3(b), we can see that with the introduction of constraints (14), (15) and (16), sensor nodes will never run out their residual energies before being charged again.

Fig. 4(a) shows the convergence of proposed Benders-based decentralized method. With the infeasibility constraint (29) and feasibility constraint (28) adding into problem (20) at steps $\{1,2,3,4\}$ and 5 respectively, the upper bound and lower bound converge quickly (takes 5 steps) to the optimal value $\Phi^{*}$. On the other hand, in each charging round, the proposed method only requires 5 times of information exchange between the $\mathrm{BS}$ and MCs. Therefore, the communication burden of MC is relatively small.

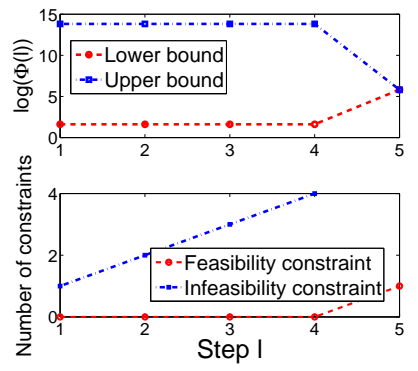

(a)

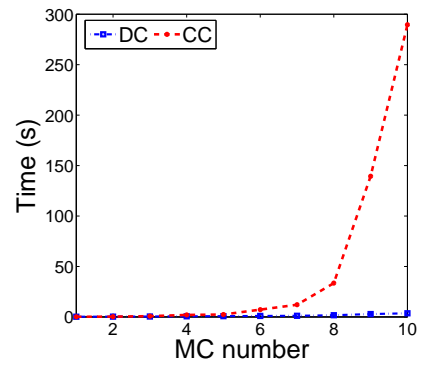

(b)
Fig. 4. a) Convergence of Benders-based decentralized method. b) Computational complexity.

Fig. 4(b) compares the computational complexity of decentralized control (DC) and centralized control (CC). We set $m \in[1,10], n=5 m$. From Fig. 4(b), we can see that DC has much lower computational complexity than $\mathrm{CC}$ : as the number of $\mathrm{MC}$ increases, the computation time of both algorithms grows; however, DC always uses much lesser computation time than CC. Although DC requires more communications than $\mathrm{CC}, \mathrm{DC}$ has much lower computational complexity.

\section{CONCLUSION}

In this paper, we proposed a decentralized method to coordinate multi-MC to fulfil the sensor charging task, with a focus on the optimal MC scheduling and charging problem to prolong the network lifetime. The goal was to keep the network operating perpetually, while enhancing the energy efficiency of MCs. This problem has been formulated as a MILP problem, which jointly optimized the MCs scheduling, the moving and charging time allocation. Furthermore, to efficiently solve this 
problem, we proposed a Benders-based decentralized method. This method decomposed the multi-MC coordination problem into several subproblems and assigned proper tasks to the BS and MCs. Through coordinating BS and MCs, as well as the parallel processing ability of MCs, we have get an optimal solution with small computing resource. The simulation results showed the convergence of proposed optimization algorithm, and the scalability due to the decentralized architecture.

\section{ACKNOWLEDGMENT}

This research is financially supported in part under Lorraine AME SATELOR project, China Postdoctoral Science Foundation 2014M551742, and NSF of China with Grant 61403340.

\section{ApPENDiX A}

\section{PROOF OF PROPOSITION 2.1}

Proof: One renewable cycle will take at most $r \varrho$ time, the reasons are two-fold: First, there are at most $n$ sensor nodes get charged in one renewable cycle (i.e., $r$ charging rounds). Second, each charging round will take at most $\varrho=\frac{e_{\max }-e_{\min }}{p_{r}}+\frac{d_{\max }}{v}$ time, where $\frac{e_{\max }-e_{\min }}{p_{r}}$ and $\frac{d_{\max }}{v}$ are the maximum charging time and traveling time, respectively. For $s_{i}$, the worst case happens when $s_{i}$ is placed at the end of the next renewable cycle, i.e., in current renewable cycle, all the sensor nodes get charged except $s_{i}$, while $s_{i}$ will be charged in the $r$ th round of the next renewable cycle. Hence, $s_{i}$ has to wait $2 r-1$ charging rounds. If $L_{i} \geq$ $(2 r-1) \varrho+\tau_{s}, s_{i}$ has enough energy to work until being charged in the next renewable cycle.

\section{APPENDIX B}

\section{PROOF OF PROPOSITION 3.1}

Proof: To determine $\theta^{l}$, we need to know how long the sensor nodes in $\left\{\mathcal{R}_{l+1}, \ldots, \mathcal{R}_{t}\right\}$ can survive after finishing the charging task in $\mathcal{R}_{l}$. Since in $\mathcal{R}_{l+1}, s_{m l+1}$ has the shortest lifetime $L_{m l+1}$, to ensure the sensor nodes in $\mathcal{R}_{l+1}$ can be charged timely, the maximum waiting time of sensor nodes in $\mathcal{R}_{l+1}$ should not exceed

$$
\varpi_{l+1}^{l}=L_{m l+1}-\tau_{l}-\frac{d_{\max }}{v} .
$$

Note that in $\mathcal{R}_{l},\left\{q_{i j}^{l}, t_{i j}^{l}, g_{i j}^{l}\right\}$ are the variables, exact $\tau_{l}$ is unknown. Since the moving and charging time of $\mathcal{R}_{l}$ will not exceed $\varrho$, we can set $\tau_{l}=\tau_{l-1}+\varrho$. Similarly, to ensure the sensor nodes in $\mathcal{R}_{l+j}, 1 \leq j \leq t-l$ can be charged before they run out their residual energies, the maximum waiting time of sensor nodes in $\mathcal{R}_{l+j}$ is

$$
\begin{aligned}
\varpi_{l+j}^{l} & =L_{m(l+j-1)+1}-\tau_{l+j-1}-\frac{d_{\max }}{v} \\
& =L_{m(l+j-1)+1}-\tau_{l-1}-\sum_{i=1}^{j+1} \varrho-\frac{d_{\max }}{v} .
\end{aligned}
$$

Note that $\varrho$ is a candidate threshold for $\theta_{l}$. To ensure sensor nodes in $\left\{\mathcal{R}_{l+1}, \ldots, \mathcal{R}_{t}\right\}$ will never fall below $e_{\text {min }}$, we can select $\theta^{l}=\min \left\{\varrho, \varpi_{l+1}^{l}, \ldots, \varpi_{t}^{l}\right\}$.

\section{APPENDIX C \\ PROOF OF THEOREM 2.1}

Proof: The proof of the Theorem 2.1 was already proved in the earlier discussion. Constraint (14) guarantees that all the sensor nodes will not run out their residual energies before they get charged again, under the condition that there is no gap between two adjacent charging rounds, which is guaranteed by the constraints (15) and (16), since $\theta_{l} \leq \varrho$.

\section{APPENDIX D}

\section{PROOF OF THEOREM 3.1}

Proof: If problem (27) has a bounded solution, in order to reduce the gap between $\Phi_{\text {lower }}$ and $\Phi_{\text {upper }}$, comparing with the previous $\Phi_{\text {lower }}, \Phi_{\text {lower }}(l+1)$ should be more close to $\Phi_{\text {upper }}$. Based on this idea, the feasible constraints added into problem (20) should satisfy:

$$
\Phi_{\text {lower }}(l+1) \geq \Phi_{\text {lower }}(v), \quad \forall v \in \mathcal{A} .
$$

Since the strong duality exists between problems (22) and (27), we have

$$
\min _{\boldsymbol{t}, \boldsymbol{g}} \Phi_{j}=\max _{\substack{\boldsymbol{\lambda}(l), \boldsymbol{\mu}(l), \boldsymbol{\alpha}(l), \boldsymbol{\beta}(l), \boldsymbol{\gamma}(l), \boldsymbol{\rho}(l), \boldsymbol{\varepsilon}(l)}} \mathcal{D}_{j} .
$$

Submitting (26) into (33) and recalling (32), we get

$$
\begin{aligned}
& \Phi_{\text {lower }}(l+1) \\
= & \sum_{i=1}^{\tilde{n}} \sum_{j=1}^{m}\left(q_{i j}(l+1) d_{i j} \epsilon+p_{j} t_{i j}(l)\right) \\
\geq & \Phi_{\text {lower }}(v) \\
= & \sum_{i=1}^{\tilde{n}} \sum_{j=1}^{m}\left(q_{i j}(l+1) d_{i j} \epsilon+p_{j} t_{i j}(v)\right) \\
= & \sum_{j=1}^{m} \min _{\boldsymbol{t}, \boldsymbol{g}} \Phi_{j} \\
= & \sum_{j=1}^{m} \max _{\boldsymbol{\beta}(l), \boldsymbol{\mu}(l), \boldsymbol{\rho}(l), \boldsymbol{\varepsilon}(l)} \mathcal{D}_{j} \\
= & \sum_{i=1}^{\tilde{n}} \sum_{j=1}^{m}\left(q_{i j}(l+1) d_{i j} \epsilon-k E_{j} q_{i j}(l+1) \mu_{i j}(v)\right. \\
& +\underline{E}_{i} q_{i j}(l+1) \alpha_{i j}(v)-\bar{E}_{i} q_{i j}(l+1) \beta_{i j}(v) \\
& \left.+q_{i j}(l+1) \frac{d_{i j}}{v} \gamma_{i j}(v)-\theta \varepsilon_{i j}(v)\right) .
\end{aligned}
$$

If problem (27) has an unbounded solution, that implies the given $\boldsymbol{q}(l)$ is conflict with problem (22). Note that the feasibility of problem (22) is related to its constraints rather than its objective function, problem (22) may be feasible when positive variables $\xi^{1}, \xi^{2}, \xi^{3}, \xi^{4}, \xi^{5}, \xi^{6}, \xi^{7}$ are introduced to relax its constraints. Hence, we construct a feasibility check problem [15] to problem (22) to validate the effectiveness of constraints:

\section{Feasibility check problem}

$$
\begin{aligned}
\underset{\boldsymbol{t}(\vartheta), \boldsymbol{g}(\vartheta), \boldsymbol{\xi}}{\min } & \mathcal{W}_{j}=\sum_{i=1}^{\tilde{n}} \sum_{k=1}^{7} \xi_{i j}^{k}, \\
\text { s.t. } \quad & \left\{\begin{array}{l}
0-\xi_{i j}^{1} \leq t_{i j}(\vartheta) \leq k E_{i} q_{i j}(l)+\xi_{i j}^{2}, \\
\left(\underline{E}_{i}-\xi_{i j}^{3}\right) q_{i j}(l) \leq p_{j} t_{i j}(\vartheta) \varsigma \leq\left(\bar{E}_{i}+\xi_{i j}^{4}\right) q_{i j}(l), \\
g_{i j}(\vartheta) \geq q_{i j}(l) \frac{d_{i j}}{v}-\xi_{i j}^{5}, \\
0-\xi_{i j}^{6} \leq t_{i j}(\vartheta)+g_{i j}(\vartheta) \leq \theta+\xi_{i j}^{7}, \\
\xi_{i j}^{1} \geq 0, \xi_{i j}^{2} \geq 0, \xi_{i j}^{3} \geq 0, \xi_{i j}^{4} \geq 0 \\
\xi_{i j}^{5} \geq 0, \xi_{i j}^{6} \geq 0, \xi_{i j}^{7} \geq 0, \forall i, \forall \vartheta \in \mathcal{B} .
\end{array}\right.
\end{aligned}
$$


From problem (35), we can see if there exist the infeasible constraints, the correspond relax variables are non-zero, while the others are zeros. Hence, $\min _{\boldsymbol{t}(\vartheta), \boldsymbol{g}(\vartheta), \boldsymbol{\xi}} \mathcal{W}_{j} \geq 0$. In order to exclude those infeasible constraints, at the $(l+1)$ th iteration, we can set:

$$
0 \geq \min _{\boldsymbol{t}(\vartheta), \boldsymbol{g}(\vartheta), \boldsymbol{\xi}} \mathcal{W}_{j}
$$

Through constructing the Lagrangian to problem (35)

$$
\begin{aligned}
\mathcal{U}_{j}= & \sum_{i=1}^{\tilde{n}}\left\{\sum_{k=1}^{7} \xi_{i j}^{k}-\left(\xi_{i j}^{1}+t_{i j}(\vartheta)\right) \lambda_{i j}(\vartheta)\right. \\
& +\left(t_{i j}(\vartheta)-k E_{i} q_{i j}(l)-\xi_{i j}^{2}\right) \mu_{i j}(\vartheta) \\
& +\left[\left(\underline{E}_{i}-\xi_{i j}^{3}\right) q_{i j}(l)-p_{j} t_{i j}(\vartheta) \varsigma\right] \alpha_{i j}(\vartheta) \\
& +\left[p_{j} t_{i j}(\vartheta) \varsigma-\left(\bar{E}_{i}+\xi_{i j}^{4}\right) q_{i j}(l)\right] \beta_{i j}(\vartheta) \\
& +\left(q_{i j}(l) \frac{d_{i j}}{v}-\xi_{i j}^{5}-g_{i j}(\vartheta)\right) \gamma_{i j}(\vartheta) \\
& -\left(\xi_{i j}^{6}+t_{i j}(\vartheta)+g_{i j}(\vartheta)\right) \rho_{i j}(\vartheta) \\
& \left.+\left(t_{i j}(\vartheta)+g_{i j}(\vartheta)-\theta-\xi_{i j}^{7}\right) \varepsilon_{i j}(\vartheta)\right\} \\
= & \sum_{i=1}^{\tilde{n}}\left[\mathcal{H}_{i j} t_{i j}(\vartheta)+\mathcal{T}_{i j} g_{i j}(\vartheta)+\left(1-\lambda_{i j}(\vartheta)\right) \xi_{i j}^{1}\right. \\
& +\left(1-\mu_{i j}(\vartheta)\right) \xi_{i j}^{2}+\left(1-\alpha_{i j}(\vartheta) q_{i j}(l)\right) \xi_{i j}^{3} \\
& +\left(1-\beta_{i j}(\vartheta) q_{i j}(l)\right) \xi_{i j}^{4}+\left(1-\gamma_{i j}(\vartheta)\right) \xi_{i j}^{5} \\
& \left.+\left(1-\rho_{i j}(\vartheta)\right) \xi_{i j}^{6}+\left(1-\varepsilon_{i j}(\vartheta)\right) \xi_{i j}^{7}+\mathcal{G}_{i j}\right]
\end{aligned}
$$

where

$$
\begin{aligned}
\mathcal{H}_{i j}= & -\lambda_{i j}(\vartheta)+\mu_{i j}(\vartheta)-p_{j} \varsigma \alpha_{i j}(\vartheta)+p_{j} \varsigma \beta_{i j}(\vartheta)-\rho_{i j}(\vartheta) \\
& +\varepsilon_{i j}(\vartheta), \\
\mathcal{T}_{i j}= & -\gamma_{i j}(\vartheta)-\rho_{i j}(\vartheta)+\varepsilon_{i j}(\vartheta), \\
\mathcal{G}_{i j}= & -k E_{i} q_{i j}(l) \mu_{i j}(\vartheta)+\underline{E}_{i} q_{i j}(l) \alpha_{i j}(\vartheta)-\bar{E}_{i} q_{i j}(l) \beta_{i j}(\vartheta) \\
& +q_{i j}(l) \frac{d_{i j}}{v} \gamma_{i j}(\vartheta)-\theta \varepsilon_{i j}(\vartheta),
\end{aligned}
$$

we can easily derive the dual problem to problem (36):

\section{Dual feasible check problem}

$$
\max _{\substack{\boldsymbol{\lambda}(\vartheta), \boldsymbol{\mu}(\vartheta), \boldsymbol{\alpha}(\vartheta), \boldsymbol{\beta}(\vartheta), \boldsymbol{\gamma}(\vartheta), \boldsymbol{\rho}(\vartheta), \boldsymbol{\varepsilon}(\vartheta)}} \sum_{i=1}^{\tilde{n}} \mathcal{G}_{i j}
$$

$$
\text { s.t. } \quad\left\{\begin{array}{l}
\mathcal{H}_{i j} \geq 0, \mathcal{T}_{i j} \geq 0, \\
1-\lambda_{i j}(\vartheta) \geq 0,1-\mu_{i j}(\vartheta) \geq 0, \\
1-\alpha_{i j}(\vartheta) q_{i j}(l) \geq 0,1-\beta_{i j}(\vartheta) q_{i j}(l) \geq 0, \\
1-\gamma_{i j}(\vartheta) \geq 0,1-\rho_{i j}(\vartheta) \geq 0,1-\varepsilon_{i j}(\vartheta) \geq 0, \forall i, \\
\boldsymbol{\lambda}(\vartheta) \geq 0, \boldsymbol{\mu}(\vartheta) \geq 0, \boldsymbol{\alpha}(\vartheta) \geq 0, \boldsymbol{\beta}(\vartheta) \geq 0, \\
\gamma(\vartheta) \geq 0, \boldsymbol{\rho}(\vartheta) \geq 0, \boldsymbol{\varepsilon}(\vartheta) \geq 0 .
\end{array}\right.
$$

Since the strong duality between problems (35) and (41), we have

$$
\min _{\boldsymbol{t}(\vartheta), \boldsymbol{g}(\vartheta), \boldsymbol{\xi}} \mathcal{W}_{j}=\max _{\substack{\boldsymbol{\lambda}(\vartheta), \boldsymbol{\mu}(\vartheta), \boldsymbol{\alpha}(\vartheta),(\vartheta) \\ \boldsymbol{\beta}(\vartheta), \boldsymbol{\gamma}(\vartheta), \boldsymbol{\rho}(\vartheta), \boldsymbol{\varepsilon}(\vartheta)}} \sum_{i=1}^{\tilde{n}} \mathcal{G}_{i j}
$$

\section{REFERENCES}

[1] A. Kurs, A. Karalis, R. Moffatt, J. D. Joannopoulos, P. Fisher, and M. Soljacic, "Wireless power transfer via strongly coupled magnetic resonances," Science, vol. 317, no. 5834, pp. 83-86, 2007.

[2] C. Wang, J. Li, F. Ye, and Y. Yang, "Improve charging capability for wireless rechargeable sensor networks using resonant repeaters," in Proceedings of IEEE ICDCS, Jun. 2015, pp. 133-142.

[3] Y. Shi, L. Xie, Y. Hou, and H. Sherali, "On renewable sensor networks with wireless energy transfer," in Proceedings of IEEE INFOCOM, Apr. 2011, pp. 1350-1358.

[4] Y. Peng, Z. Li, W. Zhang, and D. Qiao, "Prolonging sensor network lifetime through wireless charging," in Proceedings of IEEE RTSS, Nov. 2010, pp. $129-139$.

[5] C. Wang, J. Li, F. Ye, and Y. Yang, "Multi-vehicle coordination for wireless energy replenishment in sensor networks," in Proceedings of IEEE IPDPS, May 2013, pp. 1101-1111.

[6] L. He, L. Fu, L. Zheng, Y. Gu, P. Cheng, J. Chen, and J. Pan, "Esync: An energy synchronized charging protocol for rechargeable wireless sensor networks," in Proceedings of ACM MobiHoc, Aug. 2014, pp. 247-256.

[7] Y. Zhang, S. He, and J. Chen, "Data gathering optimization by dynamic sensing and routing in rechargeable sensor networks," IEEE/ACM Transactions on Networking, vol. 1, no. 99, pp. 1-15, Otc. 2015.

[8] C. Wang, J. Li, F. Ye, and Y. Yang, "Recharging schedules for wireless sensor networks with vehicle movement costs and capacity constraints," in Proceedings of IEEE SECON, Jun. 2014, pp. 468-476.

[9] L. Xie, Y. Shi, Y. Hou, W. Lou, H. Sherali, and S. Midkiff, "On renewable sensor networks with wireless energy transfer: The multi-node case," in Proceedings of IEEE SECON, Jun. 2012, pp. 10-18.

[10] L. Xie, Y. Shi, Y. T. Hou, W. Lou, and H. D. Sherali, "On traveling path and related problems for a mobile station in a rechargeable sensor network," in Proceedings of ACM MobiHoc, Jul. 2013, pp. 109-118.

[11] J. Li, C. Wang, F. Ye, and Y. Yang, "Netwrap: An ndn based real time wireless recharging framework for wireless sensor networks," in Proceedings of IEEE MASS, Oct. 2013, pp. 173-181.

[12] J. Zhao, X. Dai, and X. Wang, "Scheduling with collaborative mobile chargers inter-wsns," International Journal of Distributed Sensor Networks, vol. 2015, pp. 1-7, 2015.

[13] Z. Li, Y. Peng, W. Zhang, and D. Qiao, "J-roc: A joint routing and charging scheme to prolong sensor network lifetime," in Proceedings of IEEE ICNP, Oct. 2011, pp. 373-382.

[14] C. Hua and R. Zheng, "Robust topology engineering in multiradio multichannel wireless networks," IEEE Transactions on Mobile Computing, vol. 11, no. 3, pp. 492-503, Mar. 2012.

[15] Z. Yang, K. Long, P. You, and M. Y. Chow, "Joint scheduling of largescale appliances and batteries via distributed mixed optimization," IEEE Transactions on Power Systems, vol. 1, no. 99, pp. 1-10, 2014.

[16] S. P. Boyd and L. Vandenberghe, Convex optimization. Cambridge University Press, 2004.

[17] S. He, J. Chen, F. Jiang, D. K. Yau, G. Xing, and Y. Sun, "Energy provisioning in wireless rechargeable sensor networks," IEEE Transactions on Mobile Computing, vol. 12, no. 10, pp. 1931-1942, 2013.

[18] O. Landsiedel, K. Wehrle, and S. Götz, "Accurate prediction of power consumption in sensor networks," in Proceedings of IEEE EmNets, Apr. 2005, pp. $1-8$.

[19] J. F. Benders, "Partitioning procedures for solving mixed-variables programming problems," Numerische mathematik, vol. 4, no. 1, pp. 238-252, 1962.

Submitting (40) into (42) and recalling (36), we can get (29). 